Original Article

\title{
EFFICACY OF SEVELAMER CARBONATE IN COMBINATION WITH EDIBLE SOURCES IN HYPERPHOSPHATEMIA CONDITION ASSOCIATED WITH CHRONIC KIDNEY DISORDER
}

\author{
SHLINI P. ${ }^{*}$, DIKSHA ROY ${ }^{1}$, RICHA SINGH ${ }^{1}$ \\ 1Department of Biochemistry, Mount Carmel College, Autonomous, Palace Road Bengaluru 560052, Karnataka, India \\ *Email: shlinip1@gmail.com
}

Received: 04 May 2021, Revised and Accepted: 26 Jun 2021

\begin{abstract}
Objective: The main objective of the study was to discover a suitable dietary source of plant origin which can be recommended alongside the medication in case of hyperphosphatemia.

Methods: The 2 plant samples chow chow (Sechiumedule) and bitter gourd (Momordica carantia) and two seed samples-Flax seeds (Linumusitatissimum) and Chia seeds (Salvia hispanica) were selected for the study. The samples were extracted using methanol. The filtrate obtained was used for dialysis studies under different conditions. Inorganic phosphate was estimated in each of the conditions.

Results: The study indicated perturbation in the absorption of phosphate with the selected plant samples as talked about in the following study. The extreme concentration of phosphate among the four diverse picked plant sources was seen in bitter gourd, which was found to be 0.4686 $\mu \mathrm{moles} / \mathrm{ml}$. A minimal measure of $0.0693 \mu \mathrm{moles} / \mathrm{ml}$ of phosphate was found in chia seeds. Chow chow ended up being the best one in hyperphosphatemia condition as the amount of phosphate removed by it along with the drug, sevelamer carbonate, was calculated to be the maximum $(0.0561 \mu$ moles $)$. It was followed by chia seeds $(0.0429 \mu$ moles $)$ and flax seeds $(0.0420 \mu$ moles $)$.
\end{abstract}

Conclusion: The present study concentrates on the impact of various samples of plant extraction that can act on the phosphate absorption in the intestine by the procedure of dialysis.

Keywords: Chronic kidney disease, Hyperphosphatemia, Sevelamer carbonate

(C) 2021 The Authors. Published by Innovare Academic Sciences Pvt Ltd. This is an open access article under the CC BY license (https://creativecommons.org/licenses/by/4.0/) DOI: https://dx.doi.org/10.22159/ijcpr.2021v13i4.42742 Journal homepage: https://innovareacademics.in/journals/index.php/ijcpr

\section{INTRODUCTION}

Chronic kidney disease is actually identified as a lessened glomerular filtration rate, improved urinary albumin excretion, or perhaps both, and is actually an increasing public health problem. Hyperphosphatemia in chronic kidney disorders (CKD) individuals is actually a likely life-altering state which may result in aerobic calcification, metabolic bone disorders (renal osteodystrophy) as well as the improvement of secondary hyperparathyroidism (SHPT). Despite technology that is advanced and efficient and regular dialysis therapy the prevalence of hyperphosphatemia remains high [1].

Sevelamer carbonate, a buffered type of sevelamer hydrochloride, is actually an orally administered non-absorbed phosphate-binding anion exchange resin utilized in the healing of hyperphosphatemia wearing persistent kidney disorders (CKD). Sevelamer is available in form of capsules, tablets etc. Certain precautions have to be taken care of before and while consuming Sevelamer. This includes diet restrictions, knowing what the patient is allergic to, the record of the drugs patient is already consuming.

In the present study, we have used flax seeds, chia seeds, chow chow and bitter gourd as the different plant samples. All the chosen plant sources have a wide range of beneficial therapeutic significance. Flaxseed (Linumusitatissimum L.) is actually an oilseed used in natural and industrial health solutions. The advantages of consumption of entire flaxseed fractions including fossil oil, mucilage and protein suggest that consideration of the whole portfolio of the bioactive present is actually necessary to connect biological activity with certain compounds [2]. Chia, Salvia hispanica L., is actually a dietary and medicinal plant species used since old times by Aztec and Mayan. These seeds are a rich source of nutrition first of all of the polyunsaturated omega 3 fatty acids which protect from inflammation, improve cognitive performance and minimize the degree of cholesterol [3]. In chow chow, several pharmacological, as well as medicinal uses, are reported; it's suggested in controlled diets to decrease the health consequences related to obesity and diabetes. Chayote fruits, tuberous roots and leaves have complex carbs as dietary fiber as well as starch, minerals and vitamins [4]. Momordica charantia L. (Bitter gourd) is actually a flowering vine in the household of Cucurbitaceae. Many therapeutic qualities of the bitter gourd were studied by different researchers, like anti-diabetic, anti-ulcerogenic, anti-mutagenic, antioxidant, antitumor, anti-lipolytic, analgesic, abortifacient, anti-viral, immunomodulatory and hypoglycemic [5].

The current examination aims at discovering the suitable plant source amongst the four distinctively picked samples, which can be recommended alongside the medication in case of hyperphosphatemia.

\section{MATERIALS AND METHODS}

\section{Plant source}

Bitter Gourd (Momordica carantia) voucher specimen-DRSP012020 and Chow Chow (Sechiumedule) voucher specimen-DRSP022020 Obtained from Vasanthnagar Market, Bangalore, Karnataka, India.

Flax seeds (Linumusitatissimum) voucher specimen-DRSP032020 and Chia seeds (Salvia hispanica) voucher specimen-DRSP042020 This was obtained from Nature's basket Supermarket, Bangalore, Karnataka, India.

\section{Processing of the samples}

The 2 plant samples chow chow (Sechiumedule) and bitter gourd (Momordica carantia) that were selected were cut and sundried for $2 \mathrm{~d}$. This was finely powdered using a grinder and stored for further usage. The 2 seed samples, Flax seeds (Linumusitatissimum) and Chia seeds (Salvia hispanica) that were selected were dried in the incubator at 70 degree Celsius for $24 \mathrm{~h}$ and powdered using a grinder and stored for further usage.

\section{Drug source}

Sevelamer Carbonate: This drug was obtained from Neetha's Medicals, Bangalore, Karnataka, India. 


\section{Equipments}

The equipments used were Colorimeter (ELICO Ltd., Hyderabad, India/Model no. CL-157), Centrifuge (REMI Laboratory, Maharashtra, India), pH meter (ELICO Ltd. Hyderabad, India/Model No. LI.120), weighing balance (Shimadzu/Model No. ELB300), magnetic stirrer (REMI Laboratory, Maharashtra, India), Hot Air Oven

\section{Dialysis membrane source (Capra aegagrushircus)}

The intestine was obtained from the slaughterhouse of Bangalore mutton Stall, Vasanthnagar, Bangalore, Karnataka, India. The intestine was properly washed and cut to equal pieces before use for further analysis.

\section{Chemicals}

Methanol, Sodium Hydroxide, Hydrochloric Acid, Di-Potassium hydrogen ortho-phosphate, Calcium Carbonate, Ammonium Persulphate, Tris-HCl, Sodium Carbonate, Potassium Permanganate, Magnesium Carbonate, Sodium metabisulphite, Ammonium Molybdate were purchased from Fischer Scientific India Pvt. Ltd., Mumbai, India, Sulphuric Acid was purchased from HIMEDIA Laboratories Pvt. Lt., Gujarat, India, p-methyl amino phenol (METOL) was purchased from Loba Chemie Pvt. Ltd., Mumbai, India.

\section{Preparation of plant extract}

$10 \mathrm{~g}$ of powdered samples in $100 \mathrm{ml}$ of methanol was kept on a magnetic stirrer for $30 \mathrm{~min}$ and centrifuged at $8000 \mathrm{rpm}$ for $15 \mathrm{~min}$ at room temperature. The pellet was discarded and the supernatant was used freshly with required dilutions.

\section{Preparation of seed extract}

$10 \mathrm{~g}$ of dried samples in $100 \mathrm{ml}$ of methanol was kept on magnetic stirrer for $30 \mathrm{~min}$ and centrifuged at $8000 \mathrm{rpm}$ for $10 \mathrm{~min}$ at room temperature. The pellet was discarded and the supernatant was used freshly with required dilutions.

\section{Dialysis}

Equal portions of Capra aegagrushircus were taken. One end of each portions was tied using a twine. Hyperphosphatemia conditions were induced by adding $300 \mathrm{ppm}$ phosphate solution. Different combinations of solutions were prepared: Sevelamer Carbonate (300 ppm), Sevelamer Carbonate with phosphate solution, Phosphate with the methanolic extracts of the samples, Sevelamer carbonate along with phosphate and extracts of the 4 samples in different beakers. $1.0 \mathrm{ml}$ of different concentrations of phosphate were poured in the lumen of the intestine and dialyzed for $120 \mathrm{~min}$ and the activity of intestine was checked. Highest concentration of phosphate was poured in the lumen of the intestine and dialyzed with variation in time and activity was checked.

Similarly, $1.0 \mathrm{ml}$ of the different combinations of solutions were poured into the lumen of the intestines through the open end and was tied. This portion of the intestine now acted as a dialysis membrane. This membrane was placed in a beaker containing 30.0 $\mathrm{ml}$ MilliQ water; $1.0 \mathrm{ml}$ of the same solution was taken into a test tube to estimate the amount of phosphate before dialysis. Then this beaker was stirred time to time for $2 \mathrm{~h}$. From the eluent obtained after $120 \mathrm{~min}, 1.0 \mathrm{ml}$ was taken into a test tube to estimate the amount of phosphate after dialysis.

\section{Estimation of inorganic phosphate}

The amount of inorganic phosphate present from the eluent obtained after dialysis was measured [6].

\section{Phosphate estimation}

The same procedure was carried out for all the different combinations of solutions of Sevelamer carbonate, phosphate and sample extracts prepared.

\section{RESULTS AND DISCUSSION}

The kidneys' primary function is to filter additional water and wastes out of the blood to make urine. To keep the body working appropriately, the kidneys balance the salts and minerals, for example, calcium, phosphorus, sodium, and potassium-that circulate in the blood. Chronic kidney disorder (CKD) is longstanding disease of the kidneys leading to renal failure. As the kidneys fail to filter, wastes build up in the body. Hyperphosphatemia is an electrolyte disorder in which there is a raised degree of phosphate in the blood. Often there is low calcium levels which can bring about muscle spasms. Healthy kidneys help keep the perfect measure of phosphorus in the body. Hyperphosphatemia in chronic kidney disorder (CKD) patients is a potentially life-changing condition that can prompt cardiovascular calcification, metabolic bone ailment (renal osteodystrophy) and the development of secondary hyperparathyroidism (SHPT). It is likewise connected with the increased prevalence of cardiovascular disease and death rates.

\section{Estimation of inorganic phosphate}

\section{Standardization of inorganic phosphate}

In order to do the estimation of phosphate in the distinctive dialyzed samples with various different combinations, standardization of phosphate was carried out using potassium dihydrogen phosphate. The following graph (fig. 1) was obtained on standardization of phosphate using Fiske Subbarow method.

With the help of the graph obtained from standardization of phosphate, the amount of phosphate present in the dialyzed samples were calculated.

\section{Phosphate estimation}

Various concentrations of phosphates (in ppm) had been dialyzed to assess the activity of the intestinal membrane in the removal of the phosphates. It was noticed that there was a growth in removal of phosphates at boosting concentration (fig. 2).

Phosphate concentration of 300 ppm showed probably the highest task and was selected for additional dialysis studies in combination with the drug and extracts of the plant samples.

Examination of drug (Sevelamer carbonate) activity at the different span of dialysis

After the concentration of the phosphate to be utilized for additional dialysis research was driven, its activity together with the drug was examined at different time intervals.

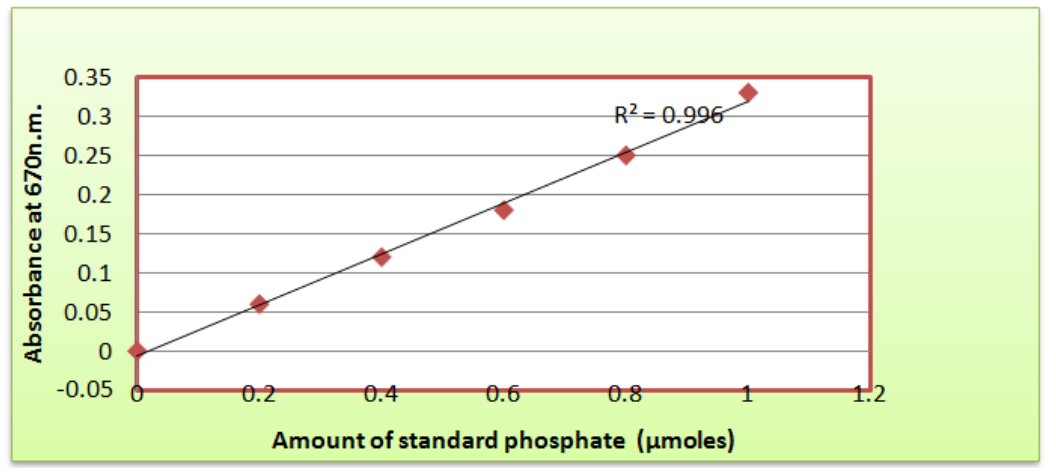

Fig. 1: Standard graph for inorganic phosphate 


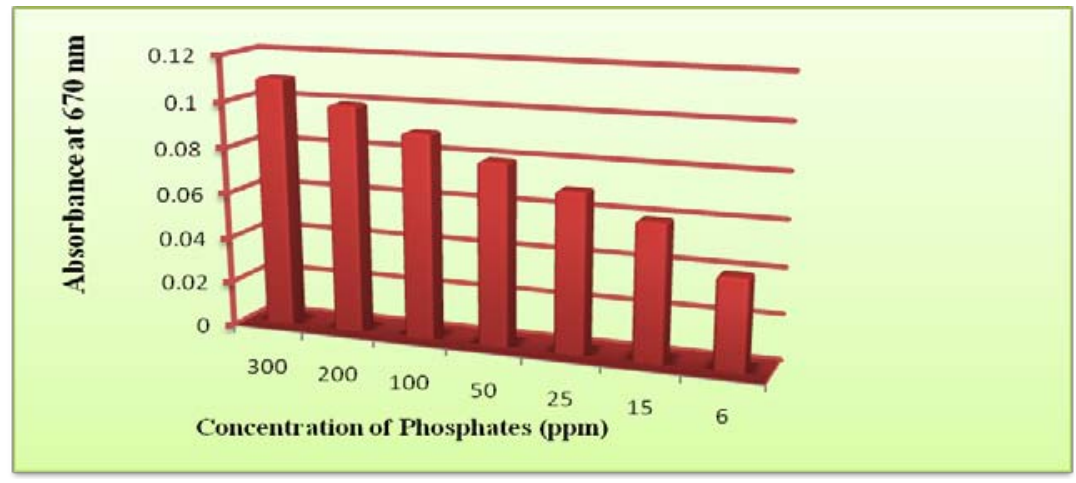

Fig. 2: Graph showing OD values for different concentrations of phosphates

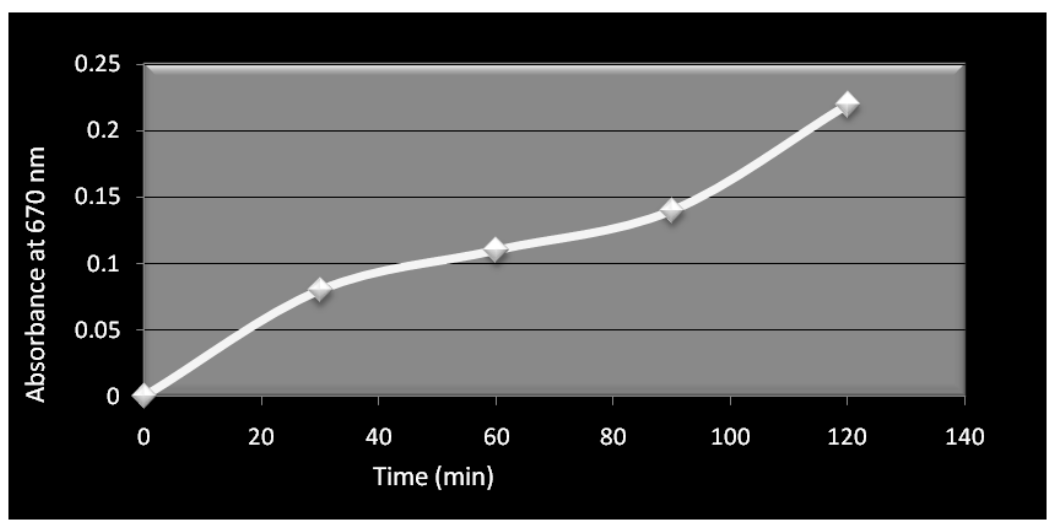

Fig. 3: Graph showing OD values of phosphate $(300 \mathrm{ppm})$ at different time intervals

The absorbance of the eluent was read after every thirty minutes for two hours. These readings demonstrated that the quantity of phosphate removed by the intestine on dialysis enhanced with an increase in dialysis time (fig. 3). A time period of two hours was driven from the received results and dialysis using various combinations of drug (Sevelamer Carbonate), phosphate (300 ppm), as well as extracts of selected plant samples, was carried out.
Estimation of phosphate concentration in the chosen plant source extracts

An assay was carried out to determine the phosphate concentration in the different selected plant extract samples. In view of the OD esteem got after dialysis (fig. 4), the concentration of phosphate was estimated utilizing the standard phosphate graph.

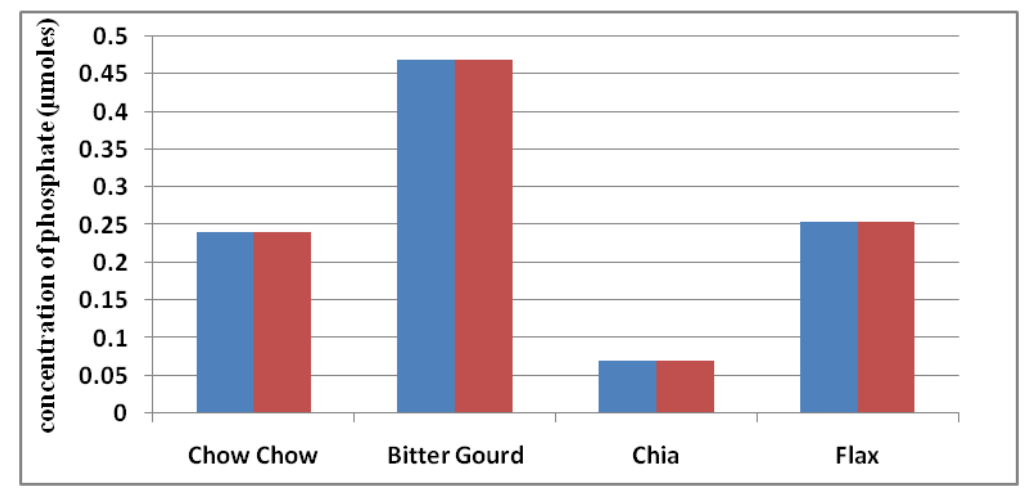

Fig. 4: Graph showing phosphate concentration ( $\mu$ moles) of different plant sources

\section{Dialysis with various plant sources after artificial induction of hyperphosphatemia}

Intestinal dialysis was done using diverse plant extracts under artificially induced hyperphosphatemia condition. No medication was used in this case. After a dialysis carried out for two hours in the intestinal membrane, the phosphate level was calculated by determining the OD values of the different samples (fig. 5).
Dialysis carried out with different plant sources along with the drug after induction of artificial hyperphosphatemia

In order to decide the best plant source that can be recommended alongside the medication (Sevelamer Carbonate), dialysis was conveyed with the plant extracts along with the medication in an induced hyperphosphatemia condition and the OD values were recorded at $670 \mathrm{~nm}$ (fig. 6). 


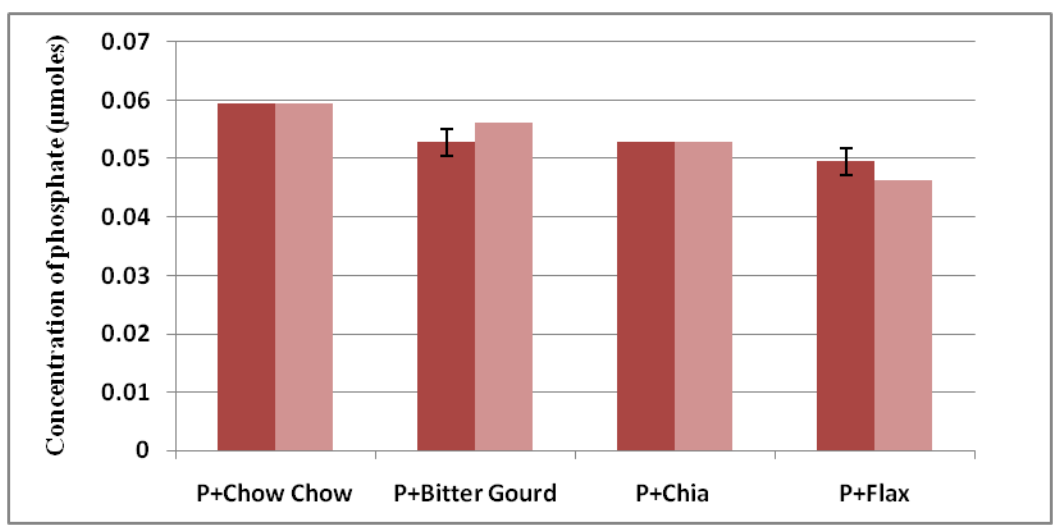

Fig. 5: Graph showing phosphate concentration ( $\mu$ moles) of different plant sources which were dialyzed along with phosphate (after a dialysis of $2 \mathrm{~h}$ )

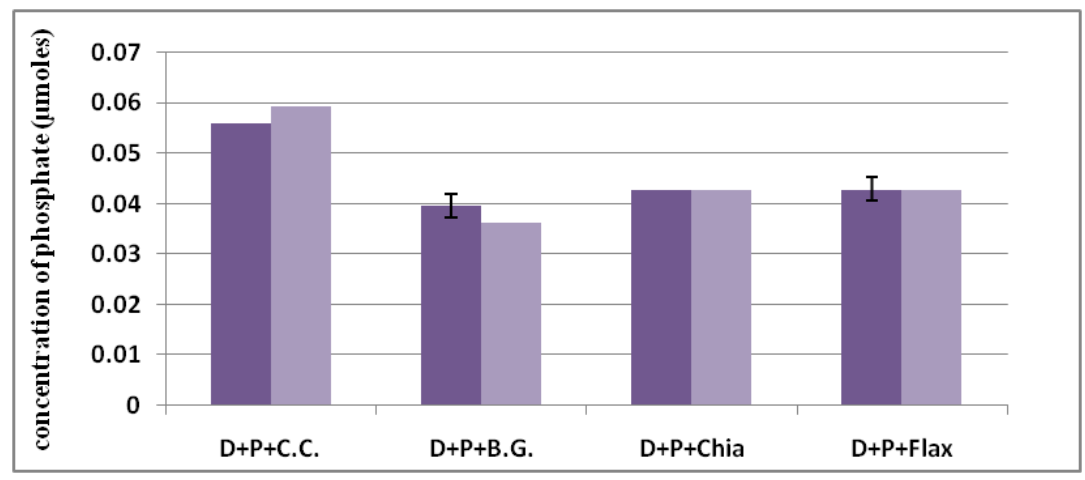

Fig. 6: Graph showing phosphate concentration ( $\mu$ moles) of different plant sources which were dialyzed along with drug and phosphate (after a dialysis of $\mathbf{2} \mathrm{h}$ )

Usually, the kidneys filtration system removes dangerous waste products as well as additional material from the blood and transforming these into urine to be passed from the entire body. If kidneys aren't operating properly-for instance, due to advanced chronic kidney disorders (kidney failure)-the kidneys might not have the ability to cleanse the blood properly. Waste products and fluid are able to build up to hazardous levels in your body (including ions). If left untreated, this could result in a number of unpleasant symptoms and ultimately be fatal. Phosphorus intake is important because both phosphorus deficiency and over-burdening impairs bone health and quality of life. Long-term excessive phosphorus loading, even if it does not cause hyperphosphatemia, can be a risk factor for cardiovascular disease (CVD) [7]. Dialysis filters out unwanted things as well as fluids from the blood before this occurs. It's a method which aids in the motion of molecules in a fix through a semi permeable membrane. Capra aegagrushircus intestine was put to be used as a membrane to hold out dialysis and then estimate the phosphate from the eluent. The most extreme measure of phosphate among the four diverse picked plant sources was seen as in bitter gourd. Minimal measure of phosphate was found in chia seeds. A dietary way to deal with phosphorus reduction is important to prevent bone impairment and CVD in patients with chronic kidney disease.

Diet assumes a significant job alongside prescription in bringing back legitimate ion concentration in the body. At the point when these plant sources were controlled alone in the hyperphosphatemia condition without the medication, sevelamer carbonate, the best source was found as chow chow (Sechium edule) trailed by bittergourd and Chia seeds. Flax seeds were seen as least compelling. Chow chow is low in calories but packed with fiber, antioxidants, and essential nutrients that make it a healthy addition to almost any diet. Chow chow contains essential vitamins and minerals. It is especially rich in vitamin $C$, which the body does not create on its own, and has to rely on external sources for. At the point when these plants sources were given alongside the medication, sevelamer, in hyperphosphatemia condition, chow chow (Sechium edule) end up being the best one followed by Chia and Flax. So, according to the investigation performed, chow chow was observed to be the best amongst the four different chosen plant sources. It bound much efficiently with the phosphate and hence mediated its removal along with sevelamer carbonate. In case of less phosphate deposition in body, it can even be recommended alone.

\section{CONCLUSION}

In chronic kidney disorder, the kidneys fail to filter wastes build up in the body. At the point when the kidneys are damaged, they can't carry out this responsibility, permitting phosphorus to develop to perilous levels in the blood. The dietary intake of phosphate-rich food is avoided during this condition as the kidneys are incapable of metabolizing them. To decrease the level of phosphate a treatment involving phosphate binders are administered to the patients. The investigation was carried out using Capra aegagrushircus intestine as a dialysis membrane in which various combinations of samples were dialysed with and without the drug. In hyperphosphatemia condition, when these plant sources were dialysed along with the drug, sevelamer carbonate, Chow (Sechium edule) proved to be the best one as it effectively lowered the phosphate concentration. The conclusion drawn from the investigation is that in state of extreme phosphate accumulation in the blood, plant sources can also be recommended along with the medication which can likewise help in the removal of phosphate efficiently from the body. If there is less accumulation of phosphate in the blood, the plant sources can even be recommended alone, with no medication.

\section{ACKNOWLEDGEMENT}

The authors wish to acknowledge the Department of Biochemistry and the management of Mount Carmel College, Autonomous, Bengaluru for offering their facilities for the analysis. 


\section{FUNDING}

Nil

\section{AUTHORS CONTRIBUTIONS}

All the authors have contributed equally.

\section{CONFLICT OF INTERESTS}

Declared none

\section{REFERENCES}

1. Kuhlmann MK. Management of hyperphosphatemia Hemodialysis Int 2006;10:338-45.

2. Shim YY, Gui B, Arnison PG, Wang Y, Reaney MJ. Flaxseed (Linumusitatissimum L.) bioactive compounds and peptide nomenclature: a review. Trends in Food Science and Technology. Elsevier Ltd; 2014.

3. De Falco B, Amato M, Lanzotti V. Chia seeds products: an overview. Phytochemistry Reviews. Springer Netherlands; 2017.

4. Angel OADC, Leon-Garcia E, Vela-Gutierrez G, De la Cruz Medina J, Garcia Varela R, Garcia HS. Chayote (Sechiumedule (Jacq.) swartz) in fruit and vegetable phytochemicals. Chem Human Health 2017;2:979-92.

5. Lim TK, Lim TK. Momordica charantia. In: Edible medicinal and non-medicinal plants; 2012. p. 331-68.

6. $\mathrm{CH}$ Fiske, Y Subbarow. The colorimetric determination of phosphorus. J Biomol Chem 1924;86:375-400.

7. Takeda E, Yamamoto H, Yamanaka Okumura H, Taketani Y. Dietary phosphorus in bone health and quality of life. Nutr Rev 2012;70:311-21. 